\title{
Modelling and Control of Autonomous under Water Vehicle using Sliding Mode Controller
}

\author{
Somu Chandra Shekar ${ }^{1}$, Basam Venkata Rao ${ }^{2}$, Prof. P. Mallikarjuna Rao ${ }^{3}$ \\ PG Scholar, Department of Electrical Engineering, Andhra University College of Engineering, Visakhapatnam, India ${ }^{1}$ \\ Scientist-D, Naval Science and Technological Laboratory (NSTL), Visakhapatnam, India ${ }^{2}$ \\ Professor, Department of Electrical Engineering, Andhra University College of Engineering, Visakhapatnam, India ${ }^{3}$
}

\begin{abstract}
This paper deals with the dynamic model and analysis of sliding mode autopilot system for an Autonomous underwater vehicle to control the motion of the AUV in 3D plane. Autonomous underwater vehicles modelling are tedious task due to its nonlinear behaviour and complex nonlinear equations. To simplify, these equations a reduced order decoupled system is considered in each plane and individual control law has been designed for each plane. The designed autopilot has been applied to AUV dynamic model and the simulations results have been presented.
\end{abstract}

Keywords: Autonomous Underwater Vehicle (AUV), Sliding Mode Control, Autopilot, MATLAB.

\section{INTRODUCTION}

The underwater vehicles can be classified as manned underwater vehicles which include submarines and atmospheric diving suit and unmanned underwater vehicles which include Remotely Operated vehicles(ROV's) and Autonomous underwater vehicles (AUV's).A remotely operated vehicle (ROV) is a tethered underwater robot designed for underwater operations. An umbilical cable transfers the required power and control signals necessary for the ROV, and receives the data from camera, sensor systems, and a manipulator arm. The manipulator arm serves the purposes of collecting small objects, cutting lines, or attaching lifting hooks for larger objects. Unlike ROV's Autonomous Underwater Vehicle's (AUV) are non-tethered. These are a self-driven through the water by a propulsion system, and contain own power supply and can maneuver in three dimensions and contain on-board computer system which is preprogrammed to determine their own path and avoid obstacles and gather information. When the objective is completed it returns to a predefined location and data can be downloaded and processed. AUV find a huge role in marine applications for many purposes such as surveying the sea bed, marine habitants, mine hunting and defense applications. When a mission is complete, the AUV will return to a pre-programmed location where the data can be downloaded and processed. The design of control system for AUV is a challenging task due to its higher order and nonlinear behavior and uncertainties in hydrodynamic coefficients and changes in center of gravity and buoyancy due to disturbances in ocean currents. These variations may lead to instability of vehicle and so we require designing a control system that makes the system robust. One practical approach to robust control design is Sliding Mode Control, which uses a nonlinear control signal to drive the states to desired switching surface. Section II describes the mathematical modelling of AUV and its decoupled Equations of motion. Section III describes the autopilot controller design and its implementation on AUV. Section IV discusses about simulation results of vehicle responses.

\section{AUV DYNAMICS}

Mathematical modelling of underwater vehicles is a difficult task as the vehicle is highly nonlinear and with little information on hydrodynamic coefficients. For deriving the equation of motion we assume that the vehicle is rigid and for the Analysis of Autonomous underwater vehicles we require two coordinate frames. These two coordinates are earth fixed and Body fixed coordinate frame. Based on SNAME the vehicle notation is tabulated as follows

Table 1. Vehicle Notation In all Degrees of Freedom

\begin{tabular}{|l|l|l|l|l|}
\hline DOF & Motion & Forces and Moments & Linear and angular velocities & Positions and Euler angles \\
\hline 1 & Surge & X & u & x \\
\hline 2 & Sway & Y & v & y \\
\hline 3 & Heave & Z & w & z \\
\hline 4 & Roll & K & p & $\emptyset$ \\
\hline 5 & Pitch & M & q & $\theta$ \\
\hline 6 & Yaw & N & r & $\psi$ \\
\hline
\end{tabular}




\section{International Journal of Innovative Research in} Electrical, Electronics, Instrumentation and Control Engineering

\section{ISO 3297:2007 Certified}

Vol. 5, Issue 6, June 2017

The transformation matrix from Body fixed to earth fixed coordinate frame is [1] as follows

$$
\left[\begin{array}{l}
\mathrm{H}_{1} \\
\mathrm{H}_{2}
\end{array}\right]=\left[\begin{array}{cc}
\mathrm{J}_{1}\left(\mathrm{H}_{2}\right) & 0 \\
0 & \mathrm{~J}_{2}\left(\mathrm{H}_{2}\right)
\end{array}\right]\left[\begin{array}{l}
\mathrm{V}_{1} \\
\mathrm{~V}_{2}
\end{array}\right]
$$

WHERE $\mathrm{H}_{1}=[\mathrm{X} \mathrm{Y} \mathrm{Z}], \mathrm{H}_{2}=[\Phi \Theta \Psi], \mathrm{V}_{1}=[\mathrm{UV} \mathrm{W}]^{\mathrm{T}}$ AND $\mathrm{V}_{2}=[\mathrm{P} \mathrm{Q} \mathrm{R}]^{\mathrm{T}}$

The generalized equations of motion in vectorial form of 6DOF [2] have been to model the vehicle as below

$$
\mathrm{M} \dot{v}+\mathrm{C}(v) v+\mathrm{D}(v) v+\mathrm{G}(\eta)=\tau
$$

where $\mathrm{M}$ is the inertia and added inertia matrix, $\mathrm{C}$ is the matrix of Coriolis and Centrifugal terms, $\mathrm{D}$ is the matrix of hydrodynamics terms, $\mathrm{G}$ is the vector of gravity and buoyant forces, $\tau$ is control-input vector describing forces and moment efforts with moveable parts of AUV according to body frame and

$$
\tau=[\mathrm{f}(\mathrm{n}), \mathrm{f}(\delta r), f(\delta s)]
$$

here $\delta s$ is the stern plane angle of $A U V, \delta r$ is the rudder angle of AUV,n is the revolution of propeller of thrusters of AUV. $v$ is the vector of linear and angular velocity of the AUV in the body-fixed coordinate frame, $\eta$ is the vector of position and attitude of the AUV in the earth fixed coordinate frame as follows

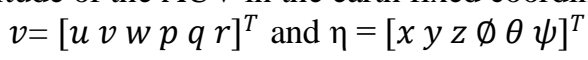

In this article we have used REMUS AUV dynamic model formed by Prestero [3]. The vehicle is of torpedo shaped and has pair of horizontal fins and a pair of vertical fins, which move together to control the attitude and orientation of the vehicle

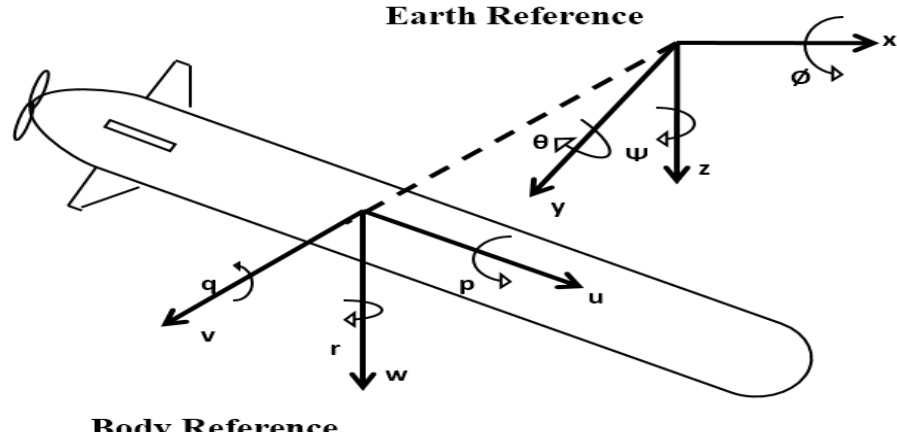

Fig 1. AUV representing reference frames

\section{A. Linearizing AUV Model}

To simplify these equations Healey and Marco suggested that 6DOF linear equations can be divided into three noninteracting subsystems for speed, steering and depth control. Each system consists of the state variables:
i. Speed system state $: \mathrm{u}(\mathrm{t})$
ii. $\quad$ Steering system states $\quad: v(t), r(t)$ and $\psi(t)$
iii. $\quad$ Diving system states $: \mathrm{w}(\mathrm{t}), \mathrm{q}(\mathrm{t}), \theta(\mathrm{t})$ and $\mathrm{z}(\mathrm{t})$

I. Forward Speed Control:

The speed control has been designed using the procedure from [4].The Equation for speed control thus becomes

$$
\left(\mathrm{m}-x_{\dot{u}}\right) \dot{u}=x_{u|u|} u|u|+x_{n|n|} n|n|
$$

II. Steering Equation of motion :

To control the sway motion of the vehicle the pitch angle as to be controlled and it is done by changing the deflection of vertical plane fins and the state space steering control model can be defined as

$$
\left[\begin{array}{ccc}
m-Y_{\dot{v}} & -Y_{\dot{r}} & 0 \\
-N_{\dot{v}} & I_{z}-N_{\dot{r}} & 0 \\
0 & 0 & 1
\end{array}\right]\left[\begin{array}{c}
\dot{v} \\
\dot{r} \\
\dot{\psi}
\end{array}\right]-\left[\begin{array}{ccc}
Y_{v} & Y_{r} & 0 \\
N_{v} & N_{r} & 0 \\
-1 & 0 & 0
\end{array}\right]\left[\begin{array}{c}
v \\
r \\
\psi
\end{array}\right]=\left[\begin{array}{c}
Y_{\delta r} \\
N_{\delta r} \\
0
\end{array}\right] \delta \mathrm{r}
$$

III. Diving Equation of motion:

Deflection in horizontal plane fins causes a change in pitch moment, thereby changing the pitch angle which changes the depth of the vehicle. 
UGC Approved Journal

IJIREEICE

\section{International Journal of Innovative Research in} Electrical, Electronics, Instrumentation and Control Engineering

\section{ISO 3297:2007 Certified}

Vol. 5, Issue 6, June 2017

So while controlling the depth of the vehicle we consider the parameters $[w, q, z, \theta]$ and all other parameters are set to zero and the state space depth control model can be defined as

$$
\left[\begin{array}{cccc}
m-Z_{\dot{w}} & -Z_{\dot{q}} & 0 & 0 \\
-M_{\dot{w}} & I_{y}-M_{\dot{q}} & 0 & 0 \\
0 & 0 & 1 & 0 \\
0 & 0 & 0 & 1
\end{array}\right]\left[\begin{array}{c}
\dot{w} \\
\dot{q} \\
\dot{Z} \\
\dot{\theta}
\end{array}\right]-\left[\begin{array}{cccc}
Z_{w} & m U+Z_{q} & 0 & 0 \\
M_{w} & M_{q} & 0 & M_{\theta} \\
1 & 0 & 0 & -U \\
0 & 1 & 0 & 0
\end{array}\right]\left[\begin{array}{c}
w \\
q \\
z \\
\theta
\end{array}\right]=\left[\begin{array}{c}
Z_{\delta s} \\
M_{\delta s} \\
0 \\
0
\end{array}\right] \delta \mathrm{s}
$$

\section{III.CONTROLLER DESIGN}

\section{A. Sliding Mode Control Design with Pole Placement}

Sliding mode control [5] is a nonlinear discontinuous control method that alters the dynamics of a system by application of a discontinuous control signal that makes the system to "slide" along a sliding surface to attain the system's normal behaviour.

The main steps involved to design a sliding-mode controller are:

1. Sliding surface design, which represents the desired dynamics

2. Design of control law, which drives the system trajectory on the sliding surface in a finite time.

For a general state space model

$$
\dot{\mathrm{x}}(\mathrm{t})=\mathrm{Ax}(\mathrm{t})+\mathrm{Bu}(\mathrm{t})
$$

Considering the pair $(\mathrm{A}, \mathrm{B})$ is controllable, the sliding surface is defined as:

$$
\sigma=s^{T} \tilde{X} \text { and } \tilde{x}=x-x_{d}
$$

Where $\mathrm{x}_{\mathrm{d}}$ is tracking desired state .Here s must be made such that the state variables converge to zero as $\mathrm{t} \rightarrow \infty$.

By the definition of Lyapunov stability theorem if

$$
\mathrm{V}(\sigma)=\frac{1}{2} \dot{\sigma} \sigma<0
$$

Then it guarantees that the error state converge to sliding surface.

Let $\dot{\sigma}=-\eta \operatorname{sign}\left(\frac{\sigma}{\varphi}\right)$ and $\eta>0$ and By differentiating the sliding surface, we get

$$
\begin{aligned}
& \dot{\sigma}=s^{\mathrm{T}} \dot{\tilde{x}}=s^{\mathrm{T}}\left(A \tilde{x}(t)+B u(t)-\dot{x}_{d}\right)=-\eta \operatorname{sign}\left(\frac{\sigma}{\varphi}\right) \\
& \text { And } u(t)=\left(s^{T} B\right)^{-1}\left(-s^{T} \dot{x}_{d}-\eta \operatorname{sign}\left(\frac{\sigma}{\varphi}\right)-s^{T} A x(t)\right)
\end{aligned}
$$

Thus $u(t)=\widehat{u}+\bar{u}$ where $\hat{u}=\left(s^{T} B\right)^{-1} s^{T} A x(t)$ is the linear feedback control and $\bar{u}=\left(s^{T} B\right)^{-1} \eta \operatorname{sign}\left(\frac{\sigma}{\varphi}\right)$ is the non linear control

And the sliding surface coefficients of $s$ are elements of left eigenvector of closed loop dynamic matrices $A_{c}$ corresponding to pole at the origin. But sliding mode suffers from chattering phenomenon which can be reduced by using sat function instead sign.

B. Controller Implementation[4]:

I. Surge motion control:

The vehicle velocity needs to be kept constant throughout the mission and should vary as desired to complete the mission. The control law[6] can be obtained as

$$
\mathrm{n}|\mathrm{n}|=\frac{1}{\mathrm{X}_{\mathrm{n}|\mathrm{n}|}}\left(\left(\mathrm{m}-\mathrm{X}_{\dot{\mathrm{u}}}\right) \mathrm{u}_{\mathrm{d}}+\mathrm{x}_{\mathrm{u}|\mathrm{u}|} \mathrm{u}|\mathrm{u}|-\left(\mathrm{m}-\mathrm{X}_{\dot{\mathrm{u}}}\right) \eta \operatorname{sat}\left(\frac{\sigma}{\varphi}\right)\right)
$$

II. Heave motion control:

A multivariable sliding mode controller[7] is used to provide accurate altitude control of the AUV. To design a sliding mode control law, we use the linearized equation discussed in the above section. 
UGC Approved Journal

IJIREEICE

Vol. 5, Issue 6, June 2017

Defining the sliding surface as $\sigma_{2}(\mathrm{t})$, the values of poles are [0 -3.4075 -3.9931+4.1887i -3.9931-4.1887i] for the system to have a peak overshoot of $0.05 \%$ and peak time of $0.75 \mathrm{sec}$. now the sliding surface is

$$
\sigma_{2}(\mathrm{t})=0.2217 \widetilde{\mathrm{w}}-0.1151 \tilde{\mathrm{q}}+0.7130 \tilde{\mathrm{z}}-0.6552 \tilde{\theta}
$$

and with $\mathrm{s}^{\mathrm{T}} \mathrm{B}=0.136$ and $\eta=1$ and $\varphi=0.2$ and the control law is

$$
\delta \mathrm{s}(\mathrm{t})=0.0411 \mathrm{w}(\mathrm{t})+0.0232 \mathrm{q}(\mathrm{t})+0.1370 \theta(\mathrm{t})-0.136 \operatorname{sign}\left(\frac{\sigma}{0.2}\right)
$$

III. Sway motion control [5] :

To control the motion in sway direction [8] it is necessary to control yaw motion since

$$
\mathrm{e}_{\mathrm{y}}=\left(\mathrm{Y}_{\mathrm{d}}-\mathrm{Y}\right) \alpha \psi_{\mathrm{d}}
$$

Using the process described in the above section we design a sliding surface to control yaw which in directly controls the motion in sway direction [9]. For this design we consider the poles to be at [-1.4 -1.45 0] which gives the sliding surface as

$$
\sigma_{3}(\mathrm{t})=0.0443 \widetilde{\mathrm{v}}-0.5004 \tilde{\mathrm{r}}+0.846 \widetilde{\psi}
$$

and with $\mathrm{s}^{\mathrm{T}} \mathrm{B}=-0.5641$ and $\eta=1$ and $\varphi=0.01$

The control law [10] is

$$
\delta \mathrm{r}(\mathrm{t})=0.2448 \mathrm{v}(\mathrm{t})-0.191 \mathrm{r}(\mathrm{t})+0.5641 \operatorname{sign}\left(\frac{\sigma}{0.01}\right)
$$

The designed control performs well on a linear model, but when applied to AUV nonlinear model, there is a great steady state error due to the absence integrator action which can be eliminated by using an integrator as shown below .Here the error in pitch direction is added with integrator in feedback

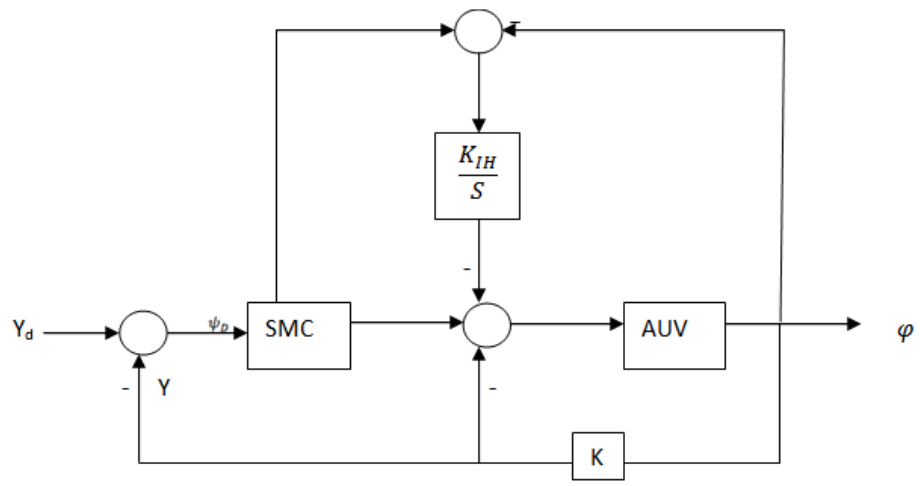

Fig 3. Block Diagram representing Integral action

\section{IV.SIMULATION RESULTS}

The above controllers have been applied to AUV model and the simulation has been conducted in MATLAB and the result shows the AUV follows the desired path successfully. Figure 4 shows the vehicle velocity is constant throughout the period with the desired command of $1.5 \mathrm{~m} / \mathrm{sec}$. Also Figure 5 and 6 shows that the vehicle responses for different commands of $Y_{d}$ is 10 meters for the first 100 seconds and 25 meters for next 100 sec. and the depth command $Z_{d}$ is 10 meters for the first $100 \mathrm{sec}$. and 25 meters for next $100 \mathrm{sec}$. The vehicle reaches the required depth and required sway motion with less error in finite time.

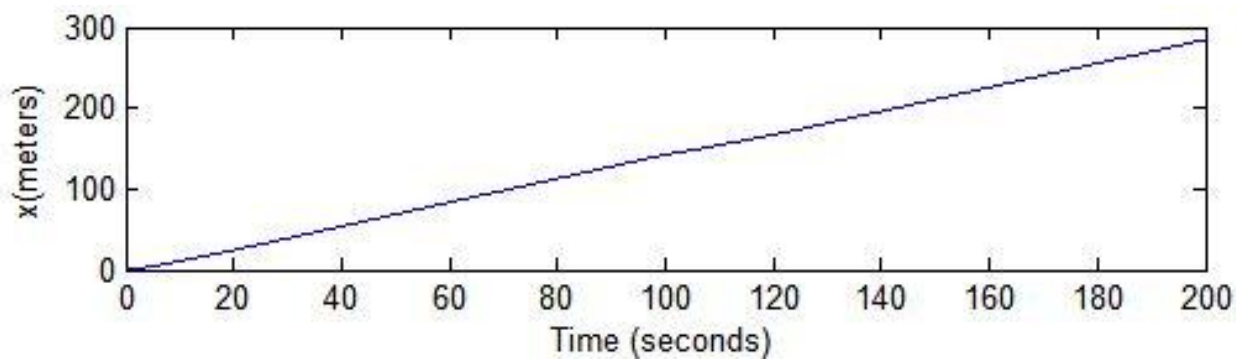

Fig 4. Trajectory of vehicle in $\mathrm{x}$ direction 
UGC Approved Journal

IJIREEICE

\section{International Journal of Innovative Research in} Electrical, Electronics, Instrumentation and Control Engineering

\section{ISO 3297:2007 Certified}

Vol. 5, Issue 6, June 2017

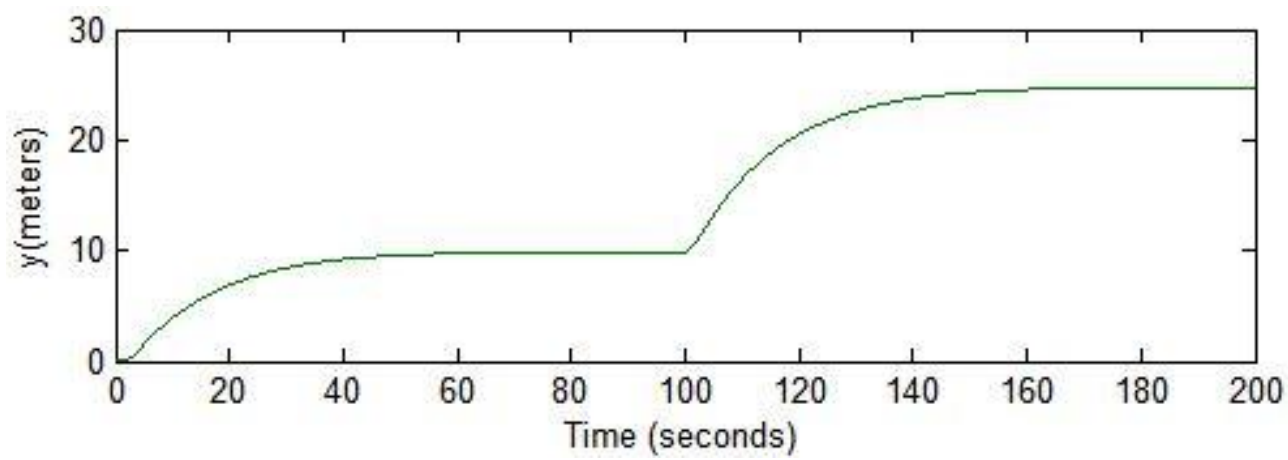

Fig 5. Trajectory of vehicle in y direction

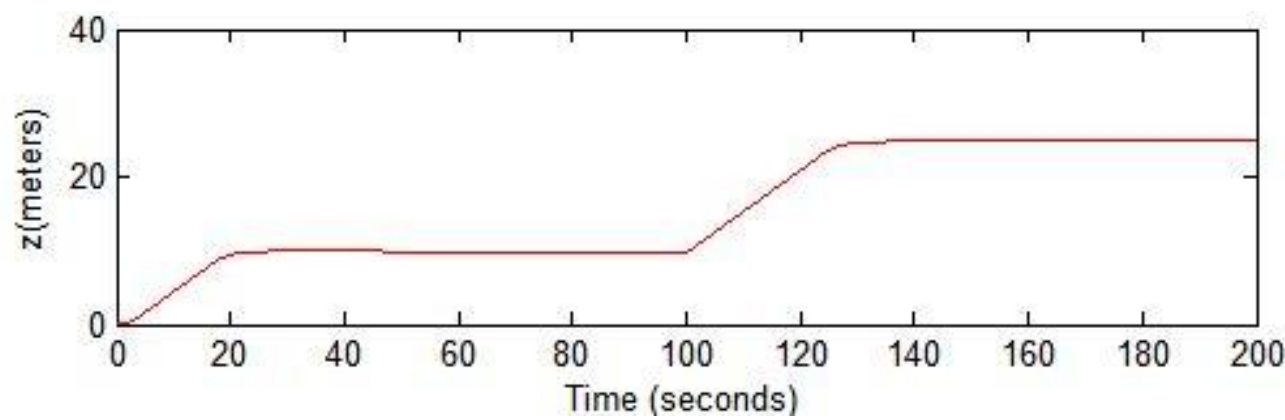

Fig 6. Trajectory of vehicle in $\mathrm{z}$ direction

\section{CONCLUSION}

This paper deals with the modelling of an AUV and to a design a control for 6 Degrees of Freedom, it has been linearized in each plane independently and sliding mode control has been proposed. The proposed autopilot control design is implemented for control of motion of an AUV in 3D plane using the decoupled equations and sliding mode control. Simulation results using MATLAB/SIMULINK have been discussed for the designed controller. Future work will be to implement the controller in real time and observe the results.

\section{REFERENCES}

[1] SNAME, Nomenclature for treating the motion of a submerged body through a fluid. Technical and Research Bulletin, 1950, pp. 1-5

[2] S. T.I. Fossen, "Guidance and Control of Ocean Vehicles", (John Wiley \& Sons, England, 1994).

[3] T. Prestero, "Verification of a Six-Degree of Freedom Simulation Model for the REMUS Autonomous Underwater Vehicle", (Master thesis, Massachusetts Institute of Technology and Woods Hole Oceanographic Institution, 2001).

[4] A. J. Healey, D. Lienard, "Multivariable sliding mode control for autonomous diving and steering of unmanned underwater vehicles", IEEE journal of oceanic engineering, vol. 18, july 1993.

[5] Juan Shi, "Design of Sliding mode Autopilot with steady state error elimination for Autonomous underwater vehicle," TENCON 2006. 2006 IEEE Region 10 Conference, pp. 1-4.

[6] “performance analysis: PID and LQR controller for remus autonomous vehicle model” IEEE Volume 3, Issue 2, July - September (2012), pp. 320-327

[7] "Depth control of autonomous underwater vehicles using indirect robust control method" Hejia Pan and Ming Xin, International Journal of Control Vol. 85, No. 1, January 2012, 98-113

[8] Nanang Syahroni, Young Bong Seo, Jae Weon Choi "Depth Control of Autonomous Underwater Vehicle Based on Open Control Platform" Proceedings of the 17th World Congress The International Federation of Automatic Control Seoul, Korea, July 6 - 11, 2008

[9] Michael J. Grimbl, Submarine depth and pitch control, ICCA, 13-16, 1993.

[10] Annaswamy, A.M., Lavretsky, E., Dydek, Z.T., Gibson, T.E. and Matsutani, M. (2013), "Recent results in robust adaptive flight control", International Journal of Adaptive Control and Signal Processing, Vol. 27 Nos 1-2, pp. 1-21. 